\title{
Le limus rédempteur de Andrej Tarkovski $^{1}$
}

\section{Paolo Mottana}

Doutor; Università degli Studi di Milano

paolo.mottana@unimib.it

\begin{abstract}
Résumé
Le cinéma de Tarkovski est riche de présences symboliques. L'essai rend, à travers le contact prolongé et fidèle aux différentes oeuvres du metteur en scène russe, la constellation de ses éléments archetipiques et de ses figures les plus significatives. Le dialogue terre-eau, le travail du feu, la récurrence de l'arbre, du bois, de l'enfance, le flux lent du temps, la phenomenologie poétique de la maison. Dans le cinéma de Tarkovski on se perd, son oeuvre, un vrai opus magnus alchimique, est un voyage dans la survivance d'un halètement mystique profondément incarné dans la matière terrestre. Son parcours circulaire, de l'arbre brûlé d'Ivan à ce qui renaît à la fin du Sacrifice miraculeusement, c'est une cheville fondamentale pour pouvoir expérimenter la puissance de l'imagination créatrice quand encore elle est l' expression d'une sensibilité inspirée à l'âme du monde".
\end{abstract}

\section{Mots clefs}

Tarkovski. Terre. Eau. Opus alchimique. Imaginal.

\section{Anamnèse imaginale}

Un quinquina d'herbe en pente et, au fond, circulaire, le miroir brumeux d'une eau immobile, peut-être un étang ou un lac. La nuque blonde d'un enfant, une femme adulte, une petite fille, um chien, une autre femme; ensuite, tous le dos tourné, ils descendent la colline alors que l'on entend, d'une voix féminine, un chant funèbre. La nostalghia prend tout d'abord la forme d'un mouvement en pente et d'une immersion entre l'herbe, l'eau et le brouillard, d'un petit groupe familial au son d'un chant féminin. C'est la descente vers cette surface qui donne un reflet et plonge, puis s'arrête tout d'un coup. Et le récit commence.

\footnotetext{
${ }^{1}$ Publicado originalmente como capítulo do livro Le regard imaginal, Editions Modulaires Européennes (2014). Revisão técnica desta versão por Danilo Fantinel, jornalista e mestrando pelo Programa de Pós-graduação em Comunicação e Informação da Universidade Federal do Rio Grande do Sul.
} 
Le dolly2 ${ }^{2}$ instrument électro-mécanique qui bascule aussi bien vers le haut que vers le bas, véritable organisme lyrique de chaque prise, remonte lentement - avec tout le temps d'un lent crescendo accordé au monde - vers le visage blond d'un enfant, des mains féminines sur ses épaules, derrière un bâtiment peu élevé, en bois, un petit étang, le long de son rivage l'homme aux cheveux noirs et le chien, dont on voit le reflet de longues rayures plus claires dans l'eau, sur un fond sombre, comme des fenêtresdans le ciel, dans l'eau du ciel. Derrière les deux figures, le petit bâtiment à nouveau, on reconnaît une Datcha. Le dolly remonte encore très lentement, il s'élève laissant sur le fond l'étang, les figures et la maison, tout autour, en rassemblant en une miniature le tableau à peine décrit, le périmètre d'une église, une abbaye médiévale s'élève, les murs très hauts avec, à l'intérieur, des fenêtres en flèche, comme des yeux et des miroirs. Lentement l'église est déjà en bas, laissant entrevoir son toit en ruines. L'église contient la maison, les figures et l'étang. Ensuite, le dolly s'interrompt, son œil reste fixé sur ce double monde où tout est retenue et débordement. Il commence a neiger sur cette abbaye et sur la Datcha, dans la campagne grise d'une Toscane abasourdie. L'atmosphère est composée, calme, silencieuse: la voix d'une femme et son chant funèbre à peine audible. Sur l'image, en surimpression, apparaissent les mots «dédié à ma mère».

Sur cette image se clôt ainsi l'un des films ${ }^{3}$ parmi les plus controversés de Andrej Tarkovski, parmi les plus pénalisés par la critique, les plus contestés par l'herméneutique«sémiologique» d'une certaine psychanalyse un peu trop hâtive et aventureuse.Peu avant cette dernière scène deux hommes meurent par l'expérience du feu. Deux hommes, ou les deux parties du même homme, un russe et un italien, viennent de passer l'un à l'autre la flamme d'une bougie en s'adonnant à un geste extrême: le premier s'est donné le feu sur une place de Rome; le deuxième a traversé une piscine thermale en se promenant, avec difficulté, avec une bougie à la faible flamme d'un rivage à l'autre, où l'eau sulfureuse n'est désormais plus, la bougie que Domenico, «homme de Dieu», lui a donnée, en

\footnotetext{
${ }^{2}$ Le Dolly: «dans les prises cinématographiques et télévisuelles, un chariot avec une petite grue qui permet à la caméra de vite se déplacer en suivant les axes horizontaux et verticaux». (DE MAURO, 2000).

3 Il s'agit de Nostalghia (1974), où l'on raconte l'affaire d'un écrivain russe, Andrej Gorçakov, qui lors de ses recherches sur un musicien, lui aussi russe, du seizième siècle, entame un parcours aux multiples significations avec une femme pour guide, Eugenia, et surtout d'un homme, Domenico, qu'il rencontre à Bagno Vignoni près de Sienne. Ce dernier est une figure complexe dont on sait que depuis sept ans il s'est renfermé dans la maison avec sa propre famille en guettant une catastrophe nucléaire. C'est de lui que Gorçakov recevra la tâche rituelle de porter une bougie allumée d'un côté à l'autre de la piscine thermale située au centre de la petite ville. Opération que le protagoniste aura effectivement accomplie à la fin du film et qui l'aura laissé sans vie, au même instant cinématographique où Domenico lui-même va s'immoler en une manifestation d'extrême protestation à Rome. Le film parvient à sa fin avec l'image que nous venons de décrire dans l'abbaye de S. Galgano à Chiusdino en Toscane. (En réalité, cette description minimale, comme d'ailleurs les autres qui suivront, ne peut que favoriser une ré-évocation du dessein essentiel de l'affaire, mais ne peut pas et ne prétend en aucune façon de rendre la densité hyper-complexe de la trame et des images dont seule la vision, et aucune narration, peut s'approcher).
} 
l'invitant à cette dernière inexplicable mission. Ils meurent tous les deux, en même temps, en parvenant ainsi au bout d'un parcours extrême et sacrificiel.Mais ce n'est que grâce à ce sacrifice, croyons-nous comprendre, que Gorçakov le protagoniste retrouve le silence de sa mémoire d'Orient dans la cathédrale italienne.

Il paraît alors que la «descente» vers l'étang de ce petit groupe familial, bloquée dans la mémoire, est maintenant compensée et transformée à un degré plus élevé. Plusieurs scissions se recomposent dans l'image de l'abbaye qui contient la Datcha: celle entre l'homme et son passé qui finalement ré-apparaît intègre dans l'amphithéâtre d'un lieu réel de l'exil: Gorçakov (chez qui il n'est pas difficile de décrypter un hétéronyme de l'auteur en exil, à cette époque, en Italie) retrouve l'enfant, la femme, la Datcha et le chien, un emblème persistant, dans le cinéma de Tarkovski, de la fidélité et de l'intégrité (et cette fois-ci c'est vraiment le chien del'auteur, son grand chien-loup réel, Dark). Et l'eau, l'étang du début, l'un des très nombreux miroirs d'eau immobile que Tarkovski aime beaucoup car, contrairement à la mer, ils renvoient au petit, au «microcosme» et à sa micro-physique (DI GIAMMATTEO, 1989, p. 17), en reflétant les moindres ondulations brisées, par le (dé)voilement, par des mouvements lents et réguliers, le code multiple du devenir, en surface et en profondeur, par le déplacement et le rayonnement. Gorçakov retrouve à la fin un coin de paix et la voix fèminine qui chantait, au début, la descente vers cette intimité familière laissée en suspens en suivant un déroulement chiastique typique de son langage poétique et cinématographique. «Entre temps, les rares premiers flocons de neige apparaissent dans l'air et, par une douceur en mouvement de danse, comme dans un rêve, ils se posent par terre». (TARKOVSKI, 1994, p. 268), sur ce paysage impossible, neige étrangère aux collines toscanes, une neige qui apaise par le don de cette conjonction imaginale. La vieille «Abbaye de Chiusdino», ruine elle aussi d'un passé médiéval peut-être éteint en Occident, contient le petit monde russe préservé dans la mémoire, comme dans un «coffret» proustien.

Il y a un lieu extrême d'une possible guérison pour cette nostalghia «mortelle», cette «compassion profonde reliée moins à sa propre privation, à son manque ou à sa séparation, qu'à la souffrance des autres dont on s'approche comme par un lien passionnel». (MASONI; VECCHI, 1997, p. 93). Parce que la nostalghiaest, en effet, un fait privé; mais, en Orient, c'est un symptôme plus profond de perception de souffrance, d'une souffrance collective, d'une souffrance du monde. Il n'est pas fortuit que Tarkovski ait entendu son propre cinéma comme une «nostalgie de l'harmonie». (BORIN, 1989, p. 113) et un tel sentiment, effectivement compris d'après cette signification, semble profondément imprégner la toile 
de son imaginaire. La nostalgie est, en effet, un fait privé, un fait vécu, payé, traversé; mais c'est aussi un sens cosmique, un avertissement pour le salut de l'homme dans le monde. Et cette nostalgie, cette «maladie du pays», du retour, de demeure, est à la recherche d'un chezsoi.

Où y a-t-il apaisement pour la nostalgie d'un monde composé, d'une intégrité et d'une fidélité à la terre, la terre natale en tant que terre originaire, en tant que terre-fonds et fondation, en tant que demeure? Tarkovski nous indique un espace religieux éclaté, lepérimètre sauvé d'une ruine de la foi qui, peut-être justement em vertu de cette ouverture et tenace persistance - Tarkovski prêtait beaucoup d'attention à l'atmosphère religieuse des lieux et des temps - est en mesure d'accueillir et de mettre à l'abri. Dans un certain sens, ce qui se consomme ici c'est une forme inédite de conjonction entre Orient et Occident, entre la «quadrature» familiale de Tarkovski - la Datcha, l'eau, la famille et le chien - une nature dense de significations personnelles mais tout aussi saturée par la densité matérielle de la terre russe, et celle d'un grand bâtiment religieux chrétien, du christianisme d'Occident maintenant brisé et consommé, mais d'autant plus impressionnant, dans cet exil ducentre, dans cette disposition, qu'il éveille d'autant plus de compassion. Chacun retrouve son propre noyau dans cet éloignement du centre, en ce point de non-retour. Ici le ciel peut alors se joindre à la terre avec pitié, non pas dans l'eau tumultueuse, mais dans la lente contagion de la neige, dans la danse rêveuse et suspendue qui fait de l'eau et du ciel un seul et unique plan en vibration. Le temps est enfin exorcisé, ou plutôt, dompté:«il neige en douceur, même si c'est le printemps. Les temps et les espaces s'approchent. Le chien fidèle les garde. Le temps ne les détruit pas, mais il se transforme en éternité: souvenir éternel, večnaja pamjat». (ŠPIDLİK, 1989, p. 20).

Tarkovski arrive ici effectivement à faire de la nostalghia une anamnesis, en joignant ce qui a été déchiré, en le recomposant, au prix de forcer les lieux et les temps. Et l'image. Extraordinaire imago eufemistica, celle-ci, du double emboîtement en tant que motif récurrent de la poétique imaginale de l'auteur et déjà presente auparavant dans le même Nostalghia, lorsqu'une autre reprise conjoignante retrouvait dans la maison de Domenico, en remontant d'une image du fleuve et des collines lointaines de la Russie dont le reflet s'imprimait sur le plancher, le bord d'une fenêtre et au-delà, les collines toscanes. Aussi pour marquer le vœu d'une ré-union, d'un rassemblement et non pas seulement pour apposer le sceau de la distance et de l'exil. L'éloignement qui «fabrique des miniatures», comme le soutient Bachelard en paraphrasant Bousquet: «l'éloignement ne disperse rien, au contraire, 
il rassemble en une miniature un pays où l'on aimerait vivre. Dans les miniatures de l'éloignement, les choses dispersées parviennent à 'se composer' et s'offrent alors à notre 'possession', en niant l'éloignement qui les a créées». (BACHELARD, 1961, p. 189).

Ce qui est à l'œuvre ici c'est un procédé d'une très grande originalité, à la fois de «miniaturisation» et d'«emboîtement»: «le schème du redoublement par emboîtements successifs nous amène directement à des procédés de 'gullivérisation', des procédés où [s'opère] le renversement des valeurs solaires symbolisés par la virilité et le gigantisme». (DURAND, 1963). La miniaturisation et l'emboîtement, procédés typiques d'«euphémisation», d'après Durand, se chargent, chez Tarkovski, de combattre la mort, la «lutte contre la putréfaction [...] et la décomposition temporelle», mais aussi celle, profondément enracinée chez le réalisateur, de retrouver le potentiel harmonisateur et le lien d'un régime nocturne puisque féminin, contre les scissions et les blessures au monde produites par la raison masculine, par son héroïsme gigantesque et schématisateur.

La charge nostalgique d'un féminin aux mille visages, présente dans le cinéma de cet auteur, est ici fortement exemplifiée aussi par la dédicace à la mère qui apparaît telle un contour biographique réel, étant donnée sa récente disparition, mais surtout par la structure des images dont la fonction est aussi de lier et tisser ensemble ce qui est décomposé et de préserver, en quelque sorte, l'instance d'une fidélité à l'origine. La grande nef de San Galgano, cependant, n'est pas un utérus qui étouffe, la datcha n'est pas qu'un retour comme régression, la Madone de l'Accouchement n'est pas, comme l'apparition de la femme enceinte le long du film, le symbole d'un impossible retour au fotus, que Gorçakov en fait s'interdit de voir, ne peut plus voir: ce que Tarkovski essaie de nous donner c'est le luxe d'une impossible re-composition, d'un instant onirique de révélation où la maison natale, l'étang, l'homme, le chien, la femme, la nature restent dans l'étreinte d'une cathédrale en ruines, oú la maison est dans une cathédrale, dans un jeu d'emboîtements au goût irréductiblement mystique plutôt que narcissique, un sens public, plutôt que privé. L'intérieur et l'extérieur, le haut et le bas apparaissent recomposés; nature, culture et foi semblent pouvoir co-habiter en ce lieu unique qui acquiert d'autant plus le goût d'unnon-où.

Esthétisme ou lyrisme gratuit y sont absents car, au contraire, Gorçakov est «fatigué de voir ces beautés excessives», comme cela est dit au début du film, justement en train de ne pas aller voir la Madone du peintre italien. ll préfère se tourner plutôt vers la trame énigmatique d'une perception interne du visible, revenir, dans un lent parcours en arrière qui passe par l'alter-ego Domenico, dans les juxtapositions et dans les passages, entre 
visages, mère-femme-amante, parmi des lieux qui débordent entre veille et sommeil, entre proche et lointain - les chambres de Domenico qui débouchent sur celles de la datcha lointaine, les collines et les eaux qui voyagent dans le temps plus que dans l'espace - en méditant les mots du père qui portent, telle une basse continue, un commentaire sur toute l'affaire:

Je me suis consumé dans la fête-bougie À l'aube vous cueillerez ma cire fondue Et là vous lirez pour qui pleurer, de quoi être hautains Comme en donnant la dernière portion de bonheur Mourir de légèreté et à l'abri d'un toit de fortune

Se donner des feux posthumes comme un mot. (MASONI, VECCHI, 1997, p. 92).

Une action aussi simple que celle de se promener avec une bougie, un symbole peutêtre de la foi en la vie éternelle, mais aussi un signe récurrent de la poétique de Tarkovski, d'un bord à l'autre d'une enveloppe d'eau chaude, d'une piscine maintenant vide, froide. Ce geste extrême de guérison, auquel Domenico confie le sort de l'humanité entière, extrême dans tous les sens parce que, tout em ayant l'entêtement enfantin d'un jeu, il est aussi sévère et rigoureux qu'un rituel de mort et renvoie, par une certaine assonance, à cette «action» qu'il faudra accomplir tous les jours à la même heure, à laquelle Alexander, dans Sacrifice ${ }^{4}$, semble donner beaucoup de confiance afin de changer quelque chose: action rituelle même dans ce dernier cas, pour autant qu'il s'agisse d'un rituel frôlant le scatologique5mais qui est néanmoins fortement liée à une eschatologie. Un tel geste, qui résonne en même temps que la mort «russe» de Domenico - étant donné qu'il n'y a qu'à l'Est que l'on connaît le sens profond du «se donner feu», comme l'auteur nous l'a appris auparavant dans le film - cette étreinte mortelle entre deux extrêmes hérésies mises en commun par l'essence symbolique radicale - au sens de la dimension du symbole à combler infiniment - de leur geste, cette

\footnotetext{
4 Dans le film (tit. orig. Offret 1986) il s'agit de l'affaire d'Alexander, jadis un acteur et aujourd'hui un intellectuel reconnu qui, dans sa maison de campagne avec sa femme, son fils Ometto frappé d'une passagère impossibilité de parler à cause d'une petite opération, et des amis, se trouve au milieu d'une soudaine urgence atomique. Au bout d'un parcours aux contours réflexifs, commémoratifs et initiatiques, Alexander arrivera à conjurer la tragédie grâce à une prière, à un vœu sacrificiel et à la rencontre sexuelle avec la femme de ménage Marie qui vit dans une église profanée. Après la rencontre, qui interrompt la menace et rend la parole à Ometto, Alexander, en profitant de l'absence temporaire des autres, donnera le feu à la maison, selon le vœu de renoncement total exprimé au moment de danger, après quoi il sera amené en ambulance. Entre temps, Ometto aura de quoi s'occuper pour arroser l'arbre sec que le père avait planté au début du film, en l'invitant à le faire tous les jours jusqu'à ce qu'il ne fleurisse miraculeusement comme cela s'était produit pour un moine japonais dont Alexander avait raconté l'histoire lors d'un monologue initial.

${ }^{5}$ L'acte en jeu que le père Alexander suggère au fils Ometto est de verser tous les jours un verre d'eau dans la tasse de la salle de bain, (presque comme si, en voulant lentement laisser bourgeonner le fond sombre et magmatique de l'eau, une eaufumier que le monde a refoulé, il se borne, justement, à évacuer). Par ailleurs, S. Galgano semble dans une certaine mesure aussi évoquer par association le caractère miraculeux de la rencontre d'Alexander avec Marie, la femme de ménage, dans l’église profanée.
} 
terrible coïncidence sacrificielle, semble être le prix nécessaire pour rendre à la Terre son lieu, dans une Abbaye profanée, un dernier «toit» découvert par une foi en l'infini.

Il y a là une leçon extraordinaire qui dit bien la souffrance de l'«accouchement» imaginal, la mythopoésie dont cette séquence de Nostalghia est une saisissante exemplification; mais combien retors et sinueux est le parcours pour produire un symbole qui puisse véritablement résumer une CEuvre aussi tenace, muette et intransigeante que celle d'un auteur tel Tarkovski, poète des images et alchimiste d'un temps qui leur est soustrait, apaisé, vrai, où l'on peut enfin ouvrir un «œil sans paupières».

\section{Le chiasme}

Tarkovski est le poète qui, lors d'un retour presque percussif et récursif des mêmes figures, presque comme s'il y avait un réseau imaginal pour aider à filtrer le temps et ses trames, fait usage le plus souvent de la figure rhétorique du chiasme. Chiasme réitéré à chaque film, presque pour coudre en une enveloppe imperméable, ou peut-être perméable à un discours ininterrompu, celui de l'Opus d'auteur, chaque élément, pour lui donner une forme en un dessein circulaire et symbolique. Ainsi le rêve d'immersion dans la nature d'Ivan près de la mère du début du film ${ }^{6}$ parvient-il à une conclusion symétrique au retour d'Ivan aux lieux de l'enfance heureuse, bien que marquée par des altérations indélébiles (l'arbre sec et brûlé). Kris Kelvin, protagoniste de Solaris ${ }^{7}$, marque son début par une apparition mise en relief par l'eau d'un étang dans la campagne opulente autour de la maison de famille; il y revient dans la dernière séquence, bien que ce soit dans un paysage plus torturé et énigmatique, désormais englouti par l'expérience vécue, et qui porte tous les signes d'une transmutation intérieure.

Outre le prologue du bégaiement d'enfance, sur cet aspect problématique du dire, qui paraît une sorte d'introduction méthodologique, les pelouses et les bois de l'enfance

\footnotetext{
${ }^{6}$ Il s'agit de L'enfance d'Ivan (tít. Orig. Ivanovo Detstvo) (1962), le film oú Ivan, garçon âgé de douze ans devenu orphelin pendant la guerre, y participe de manière active et en connaît les horreurs, en devenant ainsi un bon partisan et un expert des missions risquées. Suivi et en partie protégé par un capitaine et un lieutenant (Cholin et Galcev), il sera de toute façon emprisonné et pendu. La péripétie belliqueuse est scandée par quatre séquences oniriques assez denses où le garçon revient à son enfance de paix et d'intimité avec sa mère.

7 Film de 1972, où le psychologue Kris Kelvin est chargé d'enquêter auprès de phénomènes mystérieux censés avoir lieu sur une station dans l'orbite autour de la planète Solaris où l'on découvre une mer qui produit des effets étranges. Kelvin, après avoir pris congé du père et de sa maison, vivra une expérience complexe de rencontre avec les autres membres de la station et avec les créatures que l'océan pensant, bombardé par des rayons x, semble pouvoir extraire des profondeurs de la psyché et de la mémoire des divers et variés personnages. Kelvin rencontrera ainsi une deuxième fois sa femme morte après le suicide, avec qui il vivra une nouvelle et déchirante relation par laquelle il aura été profondément marqué. Revenu sur terre, il reviendra chez le père devant lequel il s'agenouillera alors qu'un long cadrage final nous montrera la maison paternelle et les environs comme une petite île dans l'océan de Solaris.
} 
reviennent au début et à la fin du périple autobiographique aux «mille plateaux» du miroir ${ }^{8}$. Les premières images de la maison du Stalker, avec la femme et la fille endormies, se conjoignent autour du voyage vers le Centre de la Zone du Stalker avec ses deux clients, avec la section du retour à la maison et de la scène finale où la petite fille mutante met en mouvement de manière énigmatique les verres sur la table dans Stalker ${ }^{9}$. Ce sont encore les images d'enfance, de la nature et de la maison, même lorsqu'elles sont projetées vers un parcours qui les modifie et les re-positionne, en ouvrant et fermant Nostalghia. Dans Sacrifice, l'arbre, arbre de la vie de l'Adoration des Mages de Leonardo et, tout de suite après, l'arbre sec symboliquement érigé par Alexander, revient en symétrie absolue à la fin du film - à ceci près que Andrej Rublëv $v^{10}$, film dont la structure particulièrement singulière est redoublée sur le schème à épisodes du roman de Tarkovski lui-même: la scène finale, avec le cadrage de l'île au milieu du fleuve sous la pluie sur laquelle s'arrêtent quatre chevaux - la présence du cheval est un thème récurrent de l'imaginaire tarkovskien aux fortes valeurs symboliques (FREZZATO, 1977, p. 38) - évoque une première et fulgurante apparition déjà dès le prologue, au moment de la chute du ballon: un cheval noir roulé sur le sol s'érige avec puissance, un instant avant que le cadrage ne montre le ballon par terre, dégonflé, secoué par le gaz fuyant, presque une bête blessée (peut-être une allusion à l'outrecuidance de chaque automatisation) et, tout près de lui, le cadavre du téméraire homme volant. Une allusion, peut-être, à l'impuissance de l'homme et à sa volonté de s'émanciper de son hybris, à son envie de s'éloigner de la terre où le cheval, au contraire, plonge avec plaisir.

Mais ce qui est sans doute saisissant, et qui a déjà été souligné (BORIN, 1989, p. 49; MASONI, VECCHI, 1997, p. 33) de diverses manières, c'est l'alpha-oméga, l'Azoth qui appose

\footnotetext{
${ }^{8}$ Il y a, dans ce film (tit. orig. Zerkalo) (1974) à la texture particulièrement complexe un croisement de l'histoire de l'auteur adolescent et adulte qui vit, justement, comme dans un miroir, les événements de sa famille d'origine et de celle qui est actuellement la sienne, marqués par son propre retrait du premier mariage comme par celui de la figure paternelle, et par la place centrale qu'occupe la relation avec la mère, sur un fond d'événements inquiétants de l'histoire belliqueuse et politique de ce temps-là. L'affaire est labyrinthique, marquée par une très intense élaboration des images, une traversée amoureuse approfondie par un inextricable parcours personnel récursif.

${ }^{9}$ Ce film de 1979 raconte le voyage de trois personnages - le Stalker (le Guide), l'écrivain et le scientifique -, figures à forte connotation symbolique, à l'intérieur d'un espace clôturé et interdit appelé la Zone, une sorte de structure dégradée, envahie par la nature et par l'eau, où l'on estime soit tombée une météorite ou se soit produit un phénomène étrange qui le rend impraticable. C'est ici que se trouve une Chambre des désirs, à laquelle seul les Stalkers peuvent conduire par des voies clandestines. La première et dernière parties, en dehors de la Zone, présentent, au début comme au retour du voyage, la maison et la famille du Stalker dont la fille, Scimmietta, est considérée comme une fille de la Zone, une «mutante» aux bras et jambés paralysés.

${ }^{10}$ Ce film de 1966 est la narration, scandée en neuf épisodes et un epilogue, du travail artistique, à l'époque des sanglantes guerres fratricides de 1400 em Russie, du peintre d'icônes Andrej Rublëv, acharné disciple d'harmonie, qui se dénoue entre événements au sens fortement marqué par des dimensions personnelles et historico-symboliques. Chaque épisode trace un parcours complexe où la réflexion sur l'histoire, sur la violence et sur l'opérativité artistique se laisse imbriquer avec des thèmes plus personnels et religieux. Ils débouchent enfin, après un noir et blanc prolongé, sur la vision en couleurs des icônes de Rublëv.
} 
le sceau sur l'œuvre du réalisateur russe (sans tenir compte du premier, académique mais pas moins anticipatoire long-métrage Le rouleau compresseur et le violon ${ }^{11}$ ): le raccord chiastique entre la première scène de L'enfant Ivanet la dernière du Sacrifice. L'arbre apparaît dans les deux films, l'enfant aussi, tous les deux donnent a voir la condition de l'orphelin et l'attente, la fidélité aussi obstinée que douloureuse. Arbre et Puer: deux symboles de vie et de renouveau bien qu'étant entravés, torturés et offensés. Les enfants sont tous les deux orphelins, même s'ils le sont de manière différente. L'enfant Ivan, au début du film, est filmé à travers la toile d'une araignée, peut-être pour évoquer son destin de proie, de victime, avant que la caméra ne monte en parcourant le tronc de l'arbre, de même qu'à la fin de Sacrifice la caméra remonte de l'image de l'enfant allongé jusqu'à parcourir encore le tronc d'un arbre, un arbre sec dont le «père lui avait dit qu'un jour il aurait fleuri».(TARKOVSKI, 1997, p. 321)12. L'arbre et l'enfant sont sans doute deux codes récurrents dans le cinéma de Tarkovski, et pourtant rarement ces arbres portent la caméra au ciel. Archétypes suspendus d'une tension entre homme et monde, homme et nature, esprit et matière, qui est encore invoquée et exercée, mais fondamentalement peu satisfaite. Même à la fin de Sacrifice, dans le dernier cadrage, la caméra produira, en réalité, un cadrage horizontal fidèle à l'eau et à la terre, les éléments fondamentaux du monde imaginal du cinéaste, un rez-de-chaussée qui ressemble a l'horizon possible, peut-être le plus ressenti aussi avec douleur, par Tarkovski. À la fin de L'enfance d'lvan, une fois la tragédie de l'enfant consommée dans l'histoire, une dernière insertion onirique projette un fragment d'utopie ébréchée et blessée, Ivan qui joue dans l'eau avec une petite fille, séquence qui sera, cependant, ponctuée avec insistance par le contrepoint de l'image inquiétante d'une branche tranchée d'arbre sec et brûlé, «sombre emblème brûlé de la férocité»(BORIN, 1989, p. 64).

Un arbre peint ressort au début de Sacrifice, d'une œuvre de Leonardo, une Adoration de l’Enfant quelque peu inquiétante dont Tarkovski montre les visages des Mages, peints comme des vieux hommes effrayés. La prise cinématographique parcourt le tronc

\footnotetext{
${ }^{11}$ Ce film, de 1960 (tit. orig. Katok i skripka), raconte l'histoire d’un jeune garçon nommé Saša. Moqué par les copains, il part pour aller passer un examen de violon dont le résultat, aussi à cause de la honte, sera négatif. En arrivant chez lui, l'enfant croise le chauffeur d'un rouleau compresseur, Sergej, qui l'accompagne jusqu'à sa voiture, en éveillant la jalousie de ses amis. L'enfant et le chauffeur du véhicule se retrouvent dans un entretien intense à la suite duquel Saša joue extraordinairement bien devant son nouvel ami. Le soir, les deux amis devaient se revoir pour aller au cinéma ensemble, mais la mère interdit à l'enfant d'aller au rendez-vous. Au moment où l'homme entre au cinéma avec un ami, Saša s'endorme en rêvant de monter à nouveau sur le rouleau compresseur avec Sergej.

${ }^{12}$ Cf. TARKOVSKI (1994). Le motif de l'abre fleurissant renvoie au récit qu'Alexander raconte à Ometto au début de Sacrifice et par lequel Tarkovski marque autant le sens de son travail que de son engagement artistique: «un moine, pas après pas, seau après seau portait de l'eau sur la montagne et il arrosait l'arbre séché, en croyant fermement à la nécessité de ce qu'il faisat, sans jamais abandonner, même pour un instant, la confiance en cette force miraculeuse de sa foi dans le Créateur; pour cette raison il fut témoin du Miracle: un matin les branches de l'arbre, toutes couvertes de petites feuilles, s'étaient à nouveau animées. Mais est-ce peut-être um miracle? Ce n'est que la verité». (TARKOVSKI, Sculpter..., p. 212).
} 
sanslaisser voir le petit Jésus et arrive à l'épaisse chevelure pour ensuite passer brusquement à la séquence de l'arbre sec planté dans le sable par Alexander. Peut-être qu'ici l'auteur nous parle-t-il d'une aspiration, d'une urgence d'équilibre, de composition entre ciel et terre, et entre art et vie, qui paraît toutefois toujours plus problématique -l'image est ambiguë, l'arbre est sec - et qui, dans un sens, se configure comme une tâche impossible mais pas pour autant à déserter. Cette tâche est certes menacée ou même mise en doute par des cendres de la culture de la Renaissance dont Leonardo est l'un parmi les représentants les plus importants, et avec laquelle le réalisateur a entretenu un rapport difficile en raison même de l'anthropocentrisme qu'une telle culture a contribué à promouvoir, avec ses effets néfastes, dans le monde Occidental. Effets qui se prolongent jusqu'à la menace de catastrophe qui tombe sur l'homme, menace atomique dont il sentait lui-même la présence concrète, après Hiroshima, après les explosions souterraines, après les centrales nucléaires, après Tchernobyl, événement qui aura lieu, justement, à la période des premières projections du film.

Sacrifice se trouve devant ce morceau qu'il cherche à dénouer, par une torsion radicale à une déraison de type religieux, à um douloureux abaissement, à une déposition de toute possession, de toute maitrise: Alexander aura choisi le sacrifice de soi, la totale déposition du monde dans l'espoir que cela puisse favoriser une réconciliation ou une survivance. Il choisira la voie d'une conjonction, avec la servante Maria, aux traits magiques et, en même temps, mystiques et sensuels, en parcourant à nouveau peut-être les étapes à franchir de la lutte intérieure entre foi et vie terrestre que Rublëv avait déjà traversées, en les composant à nouveau en une mémorable œuvre harmonieuse. Et pourtant, il ne semble plus y avoir d'espace, dans cette dernière œuvre, pour une mythopoïèse, pour une traduction expressive qui ne soit pas vraiment le sacrifice total, disposition dont Tarkovski avait été depuis toujours un interprète efficace, et qu'il soutenait ouvertement, comme lorsqu'il disait, en expliquant la scène initiale du Rublëv, que «la création exige d'un homme le don intégral de son être». (FREZZATO, 1977, p. 5).

\section{Nostalgie du centre}

Le chiasme est une figure circulaire qui agence et relie, qui renferme et laisse en suspens. L'œuvre entière de Tarkovski estchiasmatique, d'un film à l'autre, d'une séquence à l'autre; séquences lentes en soutien du temps jusqu'à ce que la trame naturelle soit retrouvée, jusqu'à ce que le monde soit rendu au monde. Peu de plans, beaucoup moins 
comparé à Eisenstein - comme il aimait le relever - peu de plans habités de symboles fixes et insistants, le longd'une pérégrination labyrinthique visant toutefois un Centreinvisible et inaccessible qui fonctionne tout de même comme un irrésistible aimant:c'est le sens de la «Zone» dans Stalker. Espace labyrinthique et circulaire comme l'est, à un certain moment, lastructure de la station de Solaris; comme le sont les circonvolutionsen bicyclette d'Otto, dans Sacrifice, et encore la structure même dufilm Le miroir qui renvoie les engorgements de notre esprit, commel'observe Bogani, et nous rend «nos mécanismes d'association». (BOGANI, 1989, p. 89).

Sans doute le labyrinthe formidable autour d'un lieu ou-topique, c'est justement le voyage mené à terme par le Stalker et parses clients que certains associent au «rhizome»de Deleuze et Guattari en tant que lieu où tout se défait et se compose à nouveau, en ouvrant continuellement de nouveaux accès et de nouveaux «pièges». Un tissu imprévisible et impondérable que l'on pourrait tout de même parcourir, peut-être, sous le signe de l'énigme mais aussi dans celui, interminable, de la recherche d'un Centre, d'après une récurrence anthropologique typique. Lieu soustrait dont personne semble pouvoir s'approcher, peutêtre parce qu'interdit à toute littéralité de l'appropriation? Lieu à la fois poétique et sacré, le Centre de la Zone, la légendaire «Chambre des désirs»dont on s'approche par un parcours retors et qui demande dérapage et errance, et non pas direction et impulsion; qui demande que l'on s'approche furtivement, en suivant la trace de «vagues comètes matérielles». (BORIN, 1989, p. 115), dés en métal accrochés à un ruban d'étoffe que le Stalker jette devant lui d'après une sorte de révélation artisanale. Un parcours interdisant le retour en arrière, puisqu'il n'y a pas de réversion dans aucun parcours, symbolique ou initiatique. «Zone»

\footnotetext{
Qui ne laisse pas de gardiens devant la porte du trésor et quiconfie ses héros à un guide qui ne connaît pas le chemin, à un lâche qui les envoie les premiers, exposés au risque de se fairetirer dessus, à quelqu'un qui ne décide rien par lui-même parcrainte de se tromper, à quelqu'un qui troque des allumettespour des petites pailles. Non pas à un sage et cultivé Virgile quiprofite des moments de pause et de repos pour expliquer,enseigner et entraîner; mais à un «ver» qui s'allonge dans unehumble et dévouée écoute dans le ventre de la terre, qui nerépond pas aux questions, quelqu'un qui «ne sait pas» faisanten sorte que les disciples puissent euxmêmes interroger leurspropres oracles et en interpréter les réponses. (BARIOGLIO, 2001, manuscrit inédit).
}

Un lieu enfin débarrassé de l'Homme, la «Zone» et, pour cette raison, sacré, c'est-àdire réservé au Dieu. Là où la nature, après cette sorte de Dissipatio Hurnani Generis, suit à nouveau son cours par une infiltration, par une étreinte et une macération des témoignages 
«endormis»que le parcours de l'homme aura laissés. Le désir et le dévoilement ne peuvent maintenant loger que dans le lieu déserté par l'homme. C'est peut-être le cœur de la poétique de Tarkovski, son code apophatique, si l'on peut dire, et si l'on peut dire avec Sandro Bernardi, témoignage d'une véritable «théologie négative», conscient que toute recherche de la Vérité engage l'espace du non-figurable et de l'ambiguïté du visible(BERNARDI, 1989, p. 97). La «Zone» en tant qu'espace encore inaccessible, menaçant, indéchiffrable et infiniment proche, comme cela transparaît des cadrages centraux du film. Mais inaccessible puisque «sauvé», dans son incongruité même, ruine, distante proximité. Il faut, pour s'en approcher, l'abandon de toute velléité de domination, l'abandon de toute «arme» (le pistolet de l'Écrivain, l'explosif du Scientifique). On ne peut lui rendre hommage que par le respect d'un silence, inondé par la pluie, l'abandon de toute certitude, comme cela arrive à tous les personnages du trio en route, à l'Écrivain, qui renonce enfin même à la Parole, à sa grande arme rhétorique sortie d'un coup, au Scientifique, qui laisse ses velléités bonifiantes et éclairantes, mais au fond aussi au Stalker, qui ne peut pas entrer et pour qui, malgré l'extraordinaire affaiblissement, sa descente, le Lieu n'est que le but de l'Autre.

Espace peut-être accessible à la «mutante», figure poétisante et mystérieuse, douloureuse, qui sait chanter la passion avec les mots du poète ${ }^{13}$, alors qu'elle déplace les objets dans un espace ultérieur, peut-être cet espace subtil que les «fils de la Zone»connaissent, si l'on entend, parmi ses nombreuses significations possibles, la Zone aussi et surtout en tant qu'espace d'un Monde Imaginal, en tant que lieu de réalisation nécessaire des corps subtils, comme le lieu de la Transmutation possible et infiniment interdite à tout espoir de réalisation littérale.

\section{Cosmos d'eau}

La recherche du sacré et la figuration de l'arbre sont, quoique différemment, des pôles magnétiques dans le travail de Tarkovski. Mais sa sacralitéet ses arbres sont toujours appelés vers le bas, un 'dessous' qui brouille toute orthodoxie en hétérodoxie, toute tentative de s'envoler en un enracinement plus fort à la Terre et à son limus. À ses éléments. Dans l'épaisseur imaginale ducinéma de Tarkovski, dans la rime enchaînée et récursive de ses motifs, qui semble presque augmenter et s'accumuler, film après film, qui semble

\footnotetext{
${ }^{13}$ Ce sont les mots du poète russe du dix-neuvième siècle Fedor l. Tjutcev: «j'aime tes yeux, mon amie / et leur jeu entre charme et flamme / lorsque, tout d'un coup, tu poses le regard vers le haut / et comme un éclair du ciel / tu regardes vit autour... / Mais il y a un charme encore plus intense: / Tes yeux baissés, / au moment du baiser passionné, / entre les cils mifermés / brûle le sombre feu du désir». (MASONI, 1989, p. 89).
} 
s'enrichir, même en un code symbolique commun, jusqu'à induire une sorte de syntaxe obligée, un écho de sons et de couleurs, de figures à l'inimitable énoncé, domine le code des éléments et, au centre de tout ceci, l'agrégat réitéré, obstiné, nécessaire de terre et d'eau. Terre et eau, de l'eau et de la terre mélangés, corps et âme d'un environnement brun, ocre, suie et anthracite. Mais d'eau d'abord.

De l'orage violent qui frappe Saša et Sergej, des flaques aux reflets renversés du Rouleau compresseur et le violon à la mer du dernier cadrage de Sacrifice, tout le cinéma de Tarkovski est imprégné d'eau, l'eau des fleuves, l'eau des puits et des étangs, eau qui tombe tout d'un coup, pluie interminable, violente, diffuse, déluge, eau immobile, sale, chaude, gelée, de l'eau qui pénètre à l'intérieur, dans les chambres fermées, de l'eau qui érode et envahit, dont le flux scande le temps et corrompt les objets. Il y a véritablement, chez Tarkovski, la présence permanente et déterminante d'un «psychisme hydrant» qui, au dire de Bachelard, constitue un «genre de destin», un «destin essentiel qui métamorphose infiniment la substance de l'être». (BACHELARD, 1942, p. 8-9). Et qui fait de Tarkovski luimême un «être voué à l'eau», donc un «être en vertige» qui «meurt à chaque instant», puisque «la mort quotidienne est la mort de l'eau».

Eau qui transforme et qui produit dans la matière une profondeur de mort, une mélancolie de la matière, qui la rend plus silencieuse, intense, submergée, sombre. Mais cette mort d'eau n'est pas censée être prise au sens littéral, puisqu'il s'agit plutôt d'une dissolution in anima, comme l'explique avec efficacité Hillman, en se rapportant à Héraclite, du début de l'opus:

Si nous relions les affirmations de Héraclite à propos de l'eau et de la mort à la devise alchimique bien connue - 'ne pas effectuer aucune opération jusqu'a ce que tout ne soit devenu de l'eau' - alors l'opus commence par la mort. Lorsque l'image d'un rêve est humide, cela veut dire qu'elle commence la dissolutio et devient plus absorbée dans la psyché, au sens de Bachelard, elle se transforme en âme parce que l'eau est l'élément spécifique du songe, l'élément des images réflexives et de leur flux incessant et insaisissable. Se mouiller dans les rêves renvoie au plaisir de l'âme pour sa mort, au plaisir de se laisser aller dans les flux loin des fixations et des intérêts littéralisés.(HILLMAN, 1984, p. 144).

L'eau omniprésente dans les films de Tarkovski se démarque alors d'une étroite décodification au sens maternel, et elle semble davantage renvoyer à un parcours cyclique du matériel visuel de l'auteur qui demande, pour son approfondissement in anima et son flux à travers l'élément liquide, de déborder continuellement comme cela arrive, après tout, dans les associations continues et les fade-outs de ses films, dans un aller-retour qui évoque 
sensiblement un exercice continu de solution et coagulation, entre veille et rêve entre rêverie et histoire. La matière de la mémoire, comme celle des faits, est soumise à une élaboration alchimique qui la transforme et la dilue, pour ensuite la mélanger à nouveau dans l'élément solide; puis la rendre encore humide et ainsi de suite jusqu'à ce quelle devienne matière imaginale. De l'eau au mille reflets, toutefois, qui transfigure le monde en une «solennité platonicienne», d'après l'expression de Bachelard(BACHELARD, 1942, p. 69) dans un commentaire sur Poe. Puisque l'eau, dans le reflet, idéalise le monde, en fait tomber les «bavures et les misères»; elle remplace la donnée par le «mirage»et rend enfin«ma vision». Étant à la fois surface et profondeur, l'eau permet d'opérer cette mystérieuse transmutation qui fait du littéral une vision, qui dispose un inter-monde capable de tenir ensemble l'intérieur et l'extérieur, le proche et le lointain, le personnel et l'impersonnel - et surtout le matériel et le spirituel - en une intimité sans lieu. Dans un certain sens, Borin a raison d'affirmer que «les eaux chez Tarkovski sont le reflet d'une puissante aspiration à l'Absolu à partir des reflets changeants qui émanent des mondes composés de la Terre». (BORIN, 1989, p. 24), comme il a raison d'en identifier la fonction de métaphorisation du flux temporel.

Mais l'eau c'est aussi beaucoup plus: elle est vraiment l'environnement, le milieu imaginal du cinéma de Tarkovski où rien ne peut jamais être interprété de façon rigide. Tout se dissout dans ses films: les corps, les vêtements, les maisons, les murs, les livres, les objets. L'eau fond et désagrège, aligne et corrode les corps «endormis» des objets sous l'eau, tout au long d'un fond boueux, d'après une sorte d'herméneutique liquide consciente que les choses sont destinées à se trans-fondre et à être congédiées, érodées, perdues, que l'histoire de l'homme n'est qu'une donnée transitoire. Tout est orienté vers la dé-littéralisation, tout est ramené à la profondeur, à l'ambivalence, à un caractère symbolique interminable qui fait de son cinéma un accès extraordinaire à l'expérience intérieure des choses, à l'âme du monde.

Le même océan pensant, figure comme tant d'autres jamais énigmatique du film Solaris, est certainement une «variation du motif de l'eau»; mais sa densité en fait quelque chose d'ultérieur, une matérialité impersonnelle, le symbole d'« un profond inconnaissable»possédant néanmoins des vertus de transformation et de retenue capables de donner forme aux morceaux les plus cachés, de produire de véritables corps spirituels, fantasmes et visions. On a la sensation qu'avec cette figure, et tout le long des vicissitudes qui la concernent, Tarkovski a touché un point nodal, un noyau de sa poétique même, où se croisent et entrent en tension, justement, les intentions de donner de l'espace à une 
profondeur intérieure soumise et marginalisée et celle d'une rationalité cognitive qui vise la violence et l'anéantissement, à faire disparaître - «un éclair et un souffle d'air»est la fin de Harey d'après les mots de Snaut à la fin du séjour de Kelvin à la station -chaque témoignage d'un monde autre, moitié mémoire et moitié révélation inconsciente, c'est-à-dire le limus de toute altérité qui ne soit pas que simulacre. Un noyau psychotique - mais ô combien révélateur - peut-être, face auquel Kelvin, qui est sensible à son message, qui en est touché, qui se rend disponible à l'écoute et aussi au soin amoureux de ses expressions sans avenir, à la fin il se pliera, dans un instant d'intense acceptation, sachant qu'il ne pourra pas maîtriser quelque chose qui le dépasse autant. C'est peut-être ce noyau que l'extraordinaire et dernier mouvement de caméra s'engage à exprimer en revenant progressivement en arrière, en laissant apparaître rapetissés l'étreinte à genoux du fils au père, la maison, l'étang, le bois et l'île de terre désormais déposée dans l'océan pensant de Solaris.

L'ocean de Solaris en dit beaucoup sur la conception cosmologique du réalisateur, de sa conscience de la minorité de l'homme, d'autant plus lancé vers des exploits héroïcoprométhéens qu'ils sont voués à l'échec et à la destruction. Il en dit beaucoup sur la nécessité de respecter l'inconnaissable et d'en accueillir la densité symbolique dans son indéchiffrabilité et, en même temps, dans sa nécessité de soin et d'attention. Ici aussi l'eau, qui acquiert la densité visqueuse d'un magma en rotation, ayant cessé de couler, ne représente rien d'étrange; c'est plutôt la substance plastique que l'on ne peut pas capter et qui imprègne tout voyage vers le centre, pour autant que cela soit considéré comme ésotérique, et à supposer que le voyage puisse engager la figuration d'un déplacement spatial concret. Dans un tel environnement humide, le regard de Tarkovski est capable de dissoudre toute concrétion rigide et de laisser couler la matière subtile de l'imaginaire, c'est un regard qui croise constamment la quadruple constellation de la nature des éléments, terre, feu, air, et qui en est soutenu, presque faufilé. C'est surtout un regard abaissé, rarement tourné vers le ciel qui est peut-être trop lointain parce que déserté par les hommes ou peut-être parce que Dieu a déserté le monde; un regard tourné vers la Terre, même dans le vol initial de Rublëv, qui n’a jamais la teinte du firmament, qui explore plutôt la croûte d'argile, qui la filtre soigneusement, en suit le mouvement du haut, de loin et de près pour en saisir les enchevêtrements végétaux, la fermentation, le coït perpétuel avec l'eau, comme pour réitérer continuellement une dynamique nécessaire et mystérieuse. Où un vent inconnu arrive parfois à souffler ses messages énigmatiques, pour induire crainte et doute, comme dans la «Zone», lorsque l'écrivain essaie de s'approcher de la Chambre par le 
chemin le plus court ou, comme dans le Miroir lorsque le docteur, une fois abandonnée la maison, est appelé à nouveau à se tourner vers la mère de Andrej; ou lorsque, toujours dans le même film, il va jusqu'à renverser les objets sur la table et à déchirer la nappe, soudain et inouï, venant toujours d'un ailleurs lointain.

Là où seul le feu, par moments, devient intense jusqu'à creuser le visible, tel un indice spirituel peut-être, feu aux mille apparitions fugaces et violentes ou apaisées et absorbées, petits feux et violents incendies, la petite bougie saisie par le poing de Gorçakov et le tas de cires dans la procession de la Madone de Monterchi dans Nostalghia, l'incendie de la grange à travers la pluie dans Le miroir, l'incendie impressionnant de la maison d'Alexander, à la fin de Sacrifice, les braises qui dégagent à peine de la fumée la fin de l'épisode de la cloche du Rublëv, en préparation pour la grande vision colorée des icônes.

\section{Terre-mère}

Vient ensuite la terre, la Terre Russie, la matière sombre du corps terrestre qui envahit l'écran, qui fait du cosmos un cosmos aqua-terrien (terracqueo), bas, brumeux, trouble. Terre-et-eau, terre en concurrence avec l'eau, à sa frontière qui en fait presque toujours une terre imprégnée, mouillée, terre créatrice et formatrice sur laquelle se roule le cheval noir de Rublëv au début, toute suite après la chute du ballon, scène où le réalisateur selon une autre interprétation possible de cette scène - a transposé son idée d'une dimension sacrificielle de l'CEuvre, du «don de soi», à savoir que celui qui crée est obligé de prendre des risques à chaque fois pour réaliser son rêve. Chute qui est peut-être aussi l'échec que chaque élévation prématurée ou tout détachement de la terre comporte lorsque l'on n'est pas préparé par une descente, par une écoute du monde comparable à celui que Boriska aura fait lors de l'épisode de la cloche à la fin du film. Boriska qui saura, bien qu'il ne le sache pas, du fait de l'absence d'instruction paternelle et d'un témoignage affectif du père - dont a aussi souffert le réalisateur dans son histoire personnelle - saura construire sa cloche, car c'est par amour qu'il saura administrer le savoir de la terre et des lieux. Il saura creuser la terre au bon endroit, après en avoir beaucoup récusé, et la trouvera par hasard, comme une «pierre» mise à l'écart, en tombant, en glissant encore dans le magma tellurique de la boue jusqu'au fond d'un escarpement, là où il aura trouvé le mélange nécessaire: le limus. 
Cette «pâte» dont Bachelard avait déjà rappelé qu'elle était «le schème fondamental de la matérialité», puisque «la notion même de matière est [...] strictement solidaire avec la notion de terre». (BACHELARD, 1942, p. 19). Terme que la langue italienne traduirait peutêtre par limo:

\begin{abstract}
Ce qui m'a toujours intéressé est, tout d'abord et justement, la terre. Je suis fasciné par le processus de croissance de ce qui vient de la terre, de ce qui surgit de la profondeur, les arbres, l'herbe [...] et tout est tendu vers le ciel, qui n'a pour moi aucune valeur symbolique. D'après moi, le ciel est vide et il n'y a pas que ses reflets sur la terre, dans le fleuve, dans les flaques [...] En général, j'aime la terre, je ne vois pas la boue, je vois la terre mélangée à l'eau, le limon d'où naissent les choses. (MASONI, VECCHI, 1997, p. 15).
\end{abstract}

En fait, cette argile si bien mélangée avec l'eau est le noyau de cette matière qui pourra retentir dans la forme heureuse de la cloche, symbole de la conjonction entre terre et ciel. Mais Boriska ne comprendra pas que la nature du mélange, mais aussi le lieu, le creux qui doit être créé, le poids que la structure en bois peut supporter, le temps du refroidissement. Tout ceci lui arrivera comme dans un rêve, comme dans l'effusion d'un savoir non su, mais senti ou pré-senti, comme un talent, un héritage inconscient.

Andrej Rublëv est un véritable Chant de la Terre jaillissant de partout, il est la présence centrale, comme elle le sera aussi à la fin dans le Sacrifice et comme elle l'était auparavant dans Le miroir, dont Frezzato saisit avec justesse la spécificité du paysage russe: «la violente clarté solaire, les bois épais, les sombres vallées, le sifflement du vent, les clairières ouvertes et mystérieuses, l'écume grise de l'eau, les flux majestueux et ordonnés des fleuves, où la mort n'est pas un retour soudain, mais naturel, à la 'mère humide terre'». (FREZZATO, 1977, p. 90). Il paraît toutefois vraiment réducteur, dans cette polarité eau-terre, de ne relever qu'une dominance maternelle de la «nostalgie de l'union pré-natale avec la mère»(ARGENTIERI, 1989), comme le fait Simona Argentieri dans un essai suggestif mais étonnamment dominé par un seul et unique paradigme interprétatif dans as tentative de rendre compte de la «nostalgie» tarkovskienne. Il y a, sans doute, la présence du féminin dans le cinéma et dans l'imaginaire de Tarkovski d'un féminin souvent à la connotation maternelle, et dans lequel les personnages, qui débordent aisément l'un dans l'autre, semblent être presque toujours puisés dans le monde familial et souvent même interprétés par des femmes appartenant réellement à la famille du réalisateur, sa première femme Irma dans l'Enfance d'Ivan et dans Rublëv, Larisa, sa deuxième femme, lors d'apparitions secondaires des derniers films, la mère présente dans Le miroir. Au reste, son journal témoigne largement de la volonté de proposer de tels rôles à ses proches. Et pourtant, le 
féminin présent dans son œuvre apparaît fortement transfiguré au sens symbolique, peutêtre même comme le lieu symbolique et opératif de l'opérativité artistique de Tarkovski.

Il s'agit tout d'abord d'un lieu de re-génération, d'après la leçon alchimiste, où le voyage en arrière prend la forme d'un relief ouroborique, d'un regressus ad uterum important qui modifie l'évaluation pathologisante de la psychanalyse et la ramènerait à un mythologème très répandu parmi les grandes traditions spirituelles et à l'évidente dimension archétypique: «le retour à la vie intra-utérine, dans la tradition alchimique orientale et occidentale, est une métaphore de la maîtrise du Temps et de la connaissance; il est donc un réquisit essentiel pour la conquête de l'immortalité. Ce retour en serait apparemment la via regia, grâce au fait d'avoir atteint la connaissance parfaite: l'aurea apprehensio dans les termes de l'alchimie occidentale, le jñāna d'après les termes hindous et bouddhistes; le sama rasa, c'est-à-dire le dépassement de la dualité sexuelle dans la nondualité de l'adepte, d'après les termes du tantrisme et de l'alchimie indienne». (SCHWARTZ, 2000, p. 24).

Or, si ce genre de significations n'est pas rendu explicite dans le cinéma de Tarkovski, on peut néanmoins en déduire la présence - justement - de l'envahissement d'une dimension féminine qui n'interdit pas ou ne finit pas par engloutir la création mais qui, au contraire, devient creuset imaginal, en donnant de la couleur à la matière même de l'œuvre. Le féminin tarkovskien procède de la matérialité de la Terre pour parvenir à la figuration d'un féminin parfois hiératique (la Madone de Pierre), mais beaucoup plus souvent charnel et religieux en même temps (que l'on pense aux accouplements qui se traduisent en lévitations, comme si l'unique guérison spirituelle - élévation et transformation en corps mystique - devait passer justement par la sexualité et l'abandon au féminin.

En un tel sens, le coït d'Alexander avec Maria dans Sacrifice, qui apparaît comme une lente descente vers un territoire luminal pour Alexander, territoire mystérico-orphique dont Marie est la prêtresse, se conclut avec une image qui effectivement évoque, d'après Argentieri (AGENTIERI, 1989, p. 32), la Pietà de Michel Ange; mais il ne s'agit pás d'un symbole réductible à une mort régressive, à un retour au fœus aux traits simplement narcissiques car il s'agit plutôt d'un geste sacrificiel de conjonction et de guérison. Le rituel consommé entre Alexander et Maria «sauve le monde» dans le sens où il ré-introduit la confiance et le don de soi - tous les deux étant réciproques, dans ce cas-ci - l'abaissement et la sexualité en tant que facteurs de transformation du monde (imaginal, mais aussi réel: l'enfant Ometto retrouve la parole). Dans le sens qui voit, dans le fait de s'appuyer sur un 
féminin profond, magique et mystériel, la solution au problème d'une civilisation en voie de s'acheminer apparemment vers la destruction et la raison masculine.

La poétique de Tarkovski est profondément anti-masculine en ce qu'elle se déploie contre la structure schizomorphe et schizogène de la culture, de la civilisation et de l'imaginaire masculin. Dans le cinéma de cet auteur il n'y a pas de géométrie, il n'y a pas de brusques coupures, le rythme est «fluvial», comme l'a suggéré Borin à propos des images se dissolvant les unes dans les autres, les tons sont sombres et l'imaginaire, est bien lié à l'emboîtement et à la miniaturisation, au cycle et au miroir, aux thèmes du regime nocturne et au paradigme euphémistico-synthétique de l'imaginaire. Nous sommes dans une orbite féminine, serions-nous tentés de dire, mais selon une déclinaison sophianique qui est celle de l'orient chrétien sur laquelle a raison d'insister Thomas Špidlìk. La présence du maternel dans la spiritualité russe est liée à une conception tendue vers la conciliation des opposés et à la force de la contemplation. Cette tradition mystique à laquelle Tarkovski est lié a beaucoup en commun avec celle, islamique chiîte, dont parle Corbin: c'est ici aussi qu'est invoquée une «troisième vision», au-delà des visions charnelles et spirituelles: elle consiste en une sensibilité spécifique (le «matérialisme spirituel» auquel il a souvent été fait appel pour nommer la philosophie de Tarkovski): «la tradition 'sophianique' russe appelle ce sens primordial de toutes les créatures sophia, savoir du monde, et elle le représente comme un ange divin aux formes efféminées, das ewig Weibliche». (ŠPIDLİK, 1989, p. 17).

Se peut-il alors que les mouvements érotiques en lévitation et la magie mystérieuse des transfigurations de divers personages féminins, jusqu'au mystère de Harey elle-même, puissent prendre un sens différent, et peut-être que le monde menacé par le prométhéisme, ou plutôt par le délire faustien d'un code masculin qui n'est plus contenu, puisse trouver une compensation extraordinaire dans la traduction en image de cette Sophia retrouvée? Et donc rendre le sens d'une gyno-sophia, d'un savoir du féminin, qui s'accouple avec une autre «sophia» marginalisée, celledu Puer, dont Tarkovski lui-même semble être un fervent restaurateur? Il paraît que l'espoir du réalisateur soit justement confié à ces deux codes refoulés, comme à une sorte de Philosophie de la Nature retrouvée et qui les incluait originairement, aujourd'hui franchie par les dispositifs du rationalisme et du pragmatisme.

\section{Enfants mutants}

Il n'est pas fortuit que l'enfant ou l'adolescent soit au centre de la plupart des films de Tarkovski: un enfant souvent mutilé (la «mutante» de Stalker), ou en-fant/in-fans, parce que 
bégayant ou muet (Ometto dans Sacrifice; le garçon Andrej dans le prologue du Miroir, la jeune femme muette dans Rublëv), un enfant tiraillé par les antithèses de l'expression la plus dramatique du code masculin - la guerre - (Ivan), jeune homme survivant à la recherche d'une rançon paternelle (Boriska), marginalisé, un «prisonnier», mais si riche de sensibilité et de faiblesse rédemptrice, Stalker lui-même, qui est un personnage aux traits puer et hermésiens extraordinaires. Le Puer blessé, comme le veut son archetype (HILLMAN, 1988, p. 21), instruit, dans un monde dominé par rationalité héroïque dissolvante, de la difficulté à prendre la parole, sinon par des voies de travers, métaphoriques, imaginales. Assimilable aux «béats et aux fous» dont parle Tarkovski lui-même dans Sculpter le temps à propos de Otto et Maria de Sacrifice, mais sans doute aussi de Domenico et de Rublëv luimême: pour eux «ce monde est plein de prodiges incompréhensibles en mouvement dans un monde imaginaire, pás réel. Ils ne ressemblent ni aux empiriques ni aux pragmatiques et ils ne croient pas a ce qu'ils peuvent toucher, mais il est vrai qu'ils voient dans leur imagination». (TARKOVSKI, 1997, p. 210).

D'autre part, c'est peut-être le puer Boriska qui traduit mieux que d'autres le sens de la confiance tarkovskienne dans les significations impliquées dans cet âge de la vie et la dimension cruciale de la créativité du Puer en vue d'une régénération de l'homme et du monde. Ce sera le travail innocent - parce que fondé sur un non-savoir - et orphelin-parce que articulé avec un manque, celui du père en particulier - de création de la cloche, à favoriser le retour à l'Opéra (et à la voix) de Andrej Rublëv, au terme de son renoncement aux pouvoirs, peut-être même jugés diaboliques, de la parole. Comme le rappelle Frezzato, la cloche est vraiment l'objet de la Grande CEuvre qu'est Andrej Rablëv en tant que symbole de tout imaginaire créateur, l'eau et la terre contribuant tous les deux à safusion:

D'une part, la pluie qui tombe sans arrêt sur la zone que Boriska trouve couverte de cette argile qu'il estimait être plus adaptée à une fusion couronnée par le succès; d'autre part, la matière immobile et inerte qui, secouée par le flux de l'élément d'origine céleste - une figuration des noces sacrées du ciel et de la terre - se sera prêtée, en répondant à la détermination d'une fougue acerbe et juvénile, à la matérialisation de l'opus, à la parfaite fusion de la cloche qui, de par sa position suspendue entre le ciel et la terre et de par sa forme qui la met en relation avec la voûte céleste, est un symbole d'harmonie universelle.(FREZZATO, 1997, p. 43).

Mais il n'y a pas de doute que la cloche est aussi son et giron, union de matière et voix, corps et souffle, ventre qui engendre le chant, organe poétique mais aussi le sceau d'une mise-en-commun, de fondation collective, consacrée. Et, une fois encore, comme pour la 
bénir et, selon une propension déjà notée au féminin, au moment du premier son, la caméra aura cadré d'abord une jeune femme et ensuite une femme de blanc vêtue à côté d'elle. Frezzato saisit un détail qui est loin d'être évident, c'est-à-dire une succession chromatique dans la séquence finale de l'épisode de la cloche, qui semble procéder du noir au rouge, en passant par le blanc, même s'il ne la rapporte pas explicitement à l'œuvre alchimique. L'observation est d'autant plus intéressante qu'il est certain que le cheval noir, la femme en blanc et les brasiers rouges (uniquement dans le fade-out final, puisque la scène est encore en noir et blanc), qui s'enchaînent assez vite, alors que Boriska est consolé par Andrej, ce sont les segments extrêmes qui ouvrent la voie à la contemplation des icônes de Rublëv, c'est-à-dire la médiation vers l'accès à l'opus accompli. D'une certaine façon, dans Andrej Rablëv plus que dans d'autres films, le sens de l'accomplissement est atteint, et l'effort d'un jeune Puer à se faire médiateur-Hermès de celle-ci, paraît justement. Tarkovski trouve chez le garçon, jeune dieu du foyer, capable d'extraire de la profondeur de la terre l'or de la réalisation philosophale, le véritable compagnon, le véritable auteur que le sens de l'orphelin et d'un manque profond, celui d'un monde submergé par la férocité et la destruction, lie fraternellement. À ce stade-ci de son œuvre cinématographique, l'auteur arrive peut-être à saisir le maximum de la convergence des motifs et l'or des icônes estvraiment scellé en un regard qui a abordé pour un instant la «beauté» d'une profonde renaissance créatrice. L'icône est, en fait, «beauté reconduisant au divin qui symbolise. Elle s'offre à un acte d'anamnèse du sacré par la voie de la contemplation esthétique et spirituelle». (DUBORGEL, 1991, p. 89). L'icône est le point de fusion d'une esthétique et d'une éthique de l'image qui sait figurer tout en gardant une limite d'inaccessibilité, qui se pose sur la ligne de faîte entre le visible et l'invisible. Si son fond avait été nommé «lumière», d'après les traités d'iconographie slaves, c'est parce que l'or «symbolise la lumière comme un mur de lumière, c'est-à-dire un temps comme rayonnement, bruissement de lumière et comme limite indépassable au-delà de laquelle réside le Tout autre dans sa lumière inaccessible».

Les icônes de Rublëv que Tarkovski nous montre à la fin de son film, en les parcourant de manière détaillée, en les approchant pour ensuite les dilater, entre fade-out et zoom, sont peut-être le code d'une opérativité qui assume l'image comme mystère, lieu extreme où contempler le bord de l'humain, son renvoi à un nowhere dont la médiation imaginale est néanmoins rendue possible par l'intercession de l'Ange-Imago. L'icône, fruit d'une opérativité distillée, produite par la traversée du silence et de la mort, qui a re-connu le 
féminin et la folie, qui a médité - en le dissolvant - le dogmatisme religieux et qui s'est abaissée jusqu'á pouvoir sentir le chant du mélange de terre dans toute sa précision et profondeur. C'est peut-être le point le plus lumineux de gravitation d'une poétique que l'on peut qualifier d'anti-humaniste, féminine, terrestre, mais aussi puérile et «simple», de la limpide concision d'un haïku, qu'il appréciait beaucoup, comme a pu le dire à son propos et je crois avec raison, Di Giammatteo.

Pris dans leur ensemble, ses films semblent être, en fait, très cohérents, même imbriqués les uns dans les autres; sa poésie claire, limpide comme celle du père, qu'il poursuivait peut-être tout au long de son court trajet, comme le faisait Boriska. C'est donc à um court fragment, extrait de l'une de ses conversations au contenu emblématique et à la poésie de Arsenij, qu'il parait juste de laisser la place, avec une dernière image. On congédiera ainsi, meme temporairement, ce compagnon d'ombre, ce Maître imaginal don't il est nécessaire et inévitable de négliger l'interminable et polymorphe richesse de motifs, et qui nous laisse une infinité de photogrammes, d'étincelles lumineuses où le monde nous est rendu, un monde immense, mais aussi intime, dont il reste sculptés des profils de femme, lumières soudaines, étendues d'eau sombre, argile, l'omniprésence du chien ${ }^{14}$.

Lui, il dit: 'tu sais, une fois j'étais dans la datcha, la fenêtre ouverte. L'air et l'eau entraient sur le plancher en bois, et moi je faisais en sorte qu'elle passe à l'intérieur car il y avait une flaque en train de se former dans la maison qui ressemblait à un miroir et qui donnait le reflet des choses dehors et dedans. La naissance de ce fait me plaisait sauf que le chien (Dark...) passe et naturellement, ne sachant pas qu'il y avait de l'eau, il se mouille les pattes. Il s'arrête tout de suite pour secouer les gouttes qu'il avait sur les pattes postérieures. Ensuite, le chien va dans une autre pièce alors qu'Andrej reste là à regarder la flaque. Vient le soleil, et en quelques instants, la flaque disparaît. Le chien revient, il s'arrête car il soupçonne qu'il y a encore de l'eau. Ils restent là tous les deux à regarder ce point et songent à la beauté du monde qui voit naître les choses qui disparaissent même si elles sont merveilleuses.(GUERRA, 1989, p. 65).

Un long travelling surveille mère et fils alors qu'ils marchent ensemble vers leur maison au bord du fleuve. Le garçon est pieds nus. Le chariot les accompagne

\footnotetext{
${ }^{14}$ Sur la puissance de transformation de l'œuvre de Tarkovski il ne serait pas inutille de citer un autre épisode rapporté dans Sculpter le temps. Au cours du travail autour du Miroir, le réalisateur souhaitait reconstruire la vieille maison de famille exactement telle qu'elle était lors de son enfance, mais pas seulement: à l'époque de son enfance, dans cette zone, il était possible de cultiver le sarrasin, ensuite remplacé par la luzerne cultivée et de l'avoine. Malgré la désapprobation et la méfiance des paysans de la zone, Tarkovski voulait que l'on sème à nouveau le sarrasin qui poussait luxuriant: «il s'agissait d'une démonstration des traits émotionnels particuliers de notre mémoire, de sa faculté de percer bien au-delà des voiles étendues par le temps, c'est à-dire par cela même qui devait raconter notre film. Telle était, en effet, son idée originaire. Je ne sais pas ce que serait devenu le film si le champ de sarrasin n'avait pas fleuri!... je n'ai pas de mots pour exprimer la façon dont cela était si inexplicablement important pour moi à ce temps-là. Et le champ fleurit!» (1988, p. 124). Car il ne s'agit pas de littéralisation, mais justement d'une opérativité miraculeuse de la mémoire et de la création qui ne laisse rien inchangé. Avec les paroles citées par Dostoevskij: «l'art, dit-on, doit donner le reflet de la vie etc. Ce sont des bêtises: l'écrivain (le poète) crée lui-même la vie, une vie qui, de plus, n’existait pas avant lui même dans toute sa plénitude».
} 
horizontalement, sur le fond l'eau coule vite parmi les algues d'un vert sombre. La caméra cadre pour un instant la mère, les cheveux clairs tirés, une disposition quelque peu suspendue, ensuite l'obscurité épaisse du bois, un oiseau traverse le feuillage en un éclair, la caméra fait marche arrière, un long souffle du vent, on voit la table, avec la lampe, le morceau de pain, le pichet, l'assiette, la cuillère, la nappe, le vent renverse la lampe et soulève les feuilles, les objets glissent sur le palier. L'enfant est maintenant près de l'embrasure de la porte de la maison et il fait son entrée: des tentes, des draps perforés et du linge étendu, en une floraison de voiles gonflées par le vent qui entre, sur le fond um miroir donne le reflet de la lumière violente à l'extérieur, ensuite la caméra focalise dans le miroir le visage en clair-obscur de l'enfant qui s'apprête lentement et avec incertitude à boire du lait. Tout le long de la scène et de son lent déroulement, dans le fond, les paroles d'un poème d'Arsenij Tarkovski, «Eurydice»:

L'homme n'a qu'un seul corps,

Seul comme la solitude.

L'âme est fatiguée

De cet enveloppe sans liens,

Faite d'oreilles et d'yeux,

Quatre sous de grandeur,

Et de peaux-cicatrice sur cicatrice,

Tirée sur les os.

De la cornée s'envole ainsi

Dans le puits ouvert du ciel,

Sur la route de glace,

Sur les ailes d'un oiseau,

Et entend des grilles

De sa vivante prison

Des bois et des champs chuchoter,

Le vrombissement des sept mers.

Sans le corps l'âme a honte,

Comme un corps mis à nu,

Ni pensée, ni action,

Ni projets, ni écrits.

Une énigme sans solution:

Qui revient sur ses pas,

Après avoir dansé sur la scène,

Où personne ne danse?

Et moi je rêve d'une âme

Différente, en un nouvel aspect:

Qui brûle, en passant

De la crainte à l'espoir.

Telle une flamme nourrie d'alcool,

Sans ombres en errance par la terre,

Ne laissant sur la table

Qu'un extrait de lilas comme souvenir.

Cours, enfant, ne crie pas

Sur la misérable Eurydice 


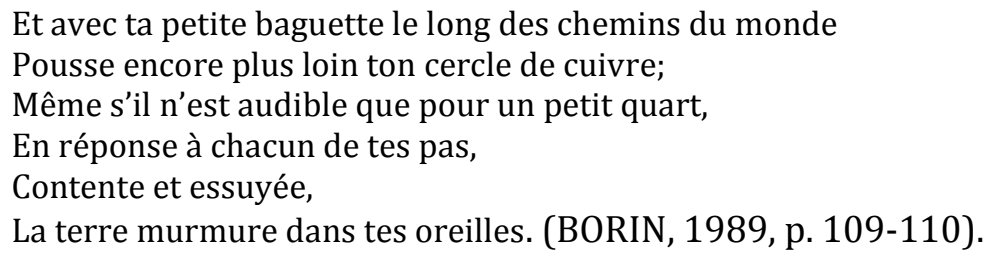

Cette âme en flammes, ce corps matériel mais subtil, «en une nouvelle apparence», qui confie à l'enfant l'espoir d'une connexion au monde, au murmure du monde terrestre, estelle peut-être le «mercure philosophique», le bout extrême d'un parcours qui rend capable d'habiter le monde? On peut se demander alors si ceci est aussi le message que ses images de feu et de sel fixent pour toujours une réflexion régie par le Puer et la Sophia.

\section{Réferènces}

ARGENTIERI, Simona. Il senso della nostalgia: da Nostalgia a Sacrificio. Paolo Zamperini, 1989.

BACHELARD, Gaston. L'eau et le rêves. Paris: Corti, 1942.

BACHELARD, Gaston. La poétique de l'espace. Paris: PUF, 1961.

BARIOGLIO, Marina. Di soppiatto in casa d'altri. 2001. [manuscrit inédit].

BERNARDI, Sandro. Fra poesía e verità. Paolo Zamperini, 1989.

BOGANI, Giovanni. Labirinti: Tarkovski, Kubrick e altri pércorsi. Paolo Zamperini, 1989.

BORIN, Fabrizio. Il cinema di Andrej Tarkovski. Rome: Jouvence, 1989.

DE MAURO, Túlio. Dizionario della língua italiana. Paravia, 2000.

DI GIAMMATTEO, Fernaldo. Uma conclusione. In: ZAMPERINI, Paolo (Ed.). Il fuoco, l'acqua, l'ombra, Andrej Tarkovij: il cinéma tra poesia e profezia. Florence: La Casa Usher, 1989.

DUBORGEL, Bruno. L’icône: art et pensée de l'invisible. Saint-Etienne: CIEREC, 1991.

DURAND, Gilbert. Les structures antropologiques de l'imaginaire. Paris: PUF, 1963.

FREZZATO, Achille. Andrej Tarkovski. Florence: La Nuova Italia, 1977.

GUERRA, Tonino. Frammenti di memoria. Paolo Zamperini, 1989.

HILLMAN, James. Il sogno e il mondo infero. Milan: Edizioni di Comunitá, 1984.

MASONI, Tullio; VECCHI, Paolo. Andrej Tarkovski. Milan: Il Castoro, 1997. 
SCHWARTZ, Arturo. L'immaginazione alchemica, ancora. Bergame: Moretti e Vitali, 2000.

ŠPIDLİK, Tomàs. Lo sfondo religioso del cinema di Tarkovski. Paolo Zamperini, 1989.

TARKOVSKI, Andrej. Racconti cinematografici. Milan: Garzanti, 1994.

\title{
O limus redentor de Andrej Tarkosvi
}

\section{Resumo}

O cinema de Tarkovski é rico em presenças simbólicas. 0 artigo traz, através do contato prolongado e fiel com as diferentes obras do diretor russo, a constelação de seus elementos arquetípicos e de suas figuras mais significativas: o diálogo terra-água, o trabalho do fogo, a recorrência da árvore, da madeira, da infância, o fluxo lento do tempo, a fenomenologia poética da casa. 0 cinema de Tarkovski é uma verdadeira opus magnum uma viagem na sobrevivência de um fôlego místico profundamente encarnado na matéria terrestre. Seu percurso circular, da árvore queimada de Ivan àquela que renasce miraculosamente no fim do sacrifício é uma cavilha fundamental para a experiência da potência da imaginação criadora quando ela é ainda expressão de uma sensibilidade inspira na alma do mundo.

\section{Palavras-chave}

Tarkovski. Terra. Água. Obra alquímica. Imaginal.

\section{The redemptive limus Andrej Tarkovski}

\begin{abstract}
The cinema of Tarkovski abounds in symbolic presences. The paper gives back, through an extended and devout contact to the various works of the Russian director, the constellation of its elements and its more meaningful figures. The dialogue earthwater, the work of the fire, the recurrence of the tree, the forest, the infancy, the slow flow of the time, the poetical phenomenology of the home. In the cinema of Tarkovski one gets lost, its work, a true alchemic opus magnus, is a travel in the survival of a mystical yearning deeply incarnated in the terrestrial matter. Its circular journery, from the burnt tree of Ivan to the tree that comes back to life miraculously at the end of the Sacrifice, is a fundamental dowel for being able to experience the power of the creative imagination when still it is the expression of an inspired sensibility to Anima Mundi.
\end{abstract}




\section{Keywords}

Tarkovski. Earth. Water. Alchemic opus. Imaginal.

Recebido em 20/10/2014

Aceito em 01/12/2014 